\title{
Difference in Risk Factors Associated with Acute Respiratory Distress Syndrome and Intubation/Death in Patients with Coronavirus Disease 2019: A Two- hospital Retrospective Cohort Study in Hubei, China
}

Jing-Lan Mu ( $\nabla$ mujinglanbb@gmail.com )

Drum Tower Hospital, Medical Department of Nanjing University

Ming-Zhe Qin

General Hospital of Central Theatre Command of PLA

Hui-Mei Sun

Nanfang Hospital, Southern Medical University

\section{Bao-Sheng Guo}

Drum Tower Hospital, Medical Department of Nanjing University

Shi-Da Qiu

Nanfang Hospital, Southern Medical University

Fu Zhang

Nanfang Hospital, Southern Medical University

Huang Yang

Nanfang Hospital, Southern Medical University

Juan Nie

The People's Hospital of Honghu

Xu-Dong Liu

Sun Yat-sen University

\section{Wing-Fai Yeung}

The Hong Kong Polytechnic University, Hong Kong

Xiao-Dong Liu

The Chinese University of Hong Kong, Hong Kong

Ye-Hong Dong

Nanfang Hospital, Southern Medical University

Bi-Xi Li

General Hospital of Central Theatre Command of PLA

Xiao-Yang Song

General Hospital of Central Theatre Command of PLA

Shao-Yan Lin

Nanfang Hospital, Southern Medical University

Ke-Xuan Liu

Nanfang Hospital, Southern Medical University 
Research Article

Keywords: COVID-19, acute respiratory distress syndrome, intubation or death, initial intubation time

Posted Date: April 20th, 2021

DOI: https://doi.org/10.21203/rs.3.rs-397008/v1

License: (9) (1) This work is licensed under a Creative Commons Attribution 4.0 International License. Read Full License 


\section{Abstract}

Background: The reviews on the risk factors with ARDS and the worse outcomes concluded lacking robust data of risk factors to prevent COVID-19 and identified an urgent need for large sample and high-quality research in this area, as well as the features of the ARDS.

Methods: This retrospective cohort study included 333 COVID-19 inpatients at two hospitals in Hubei of China in 2020. The COVID-19-related ARDS was diagnosed according to the Berlin criteria. The outcomes were ARDS development and the intubation or in-hospital death. The cox proportional hazard ratio (HR) models were employed to determine the significant risk factors.

Results: The median number of days from symptom onset to ARDS diagnosis was 11.0 (IQR, 8.0-13.0). Up to 84.1\% COVID-19-related ARDS patients demonstrated multiple organ injuries. The mortality rates were $41.9 \%$ and $85.7 \%$ in moderate and severe ARDS. The survival patients on invasive mechanical ventilation (IMV) had been intubated earlier since ARDS diagnosis than those who had not survived (5.5 median days, IQR 4.0-7.0 days versus 11.5 median days, IQR 6.0-14.0 days, $\mathrm{P}<0.001)$. Males and all abnormal laboratory indices associated with the higher risk of ARDS $(P<0.05)$ but were not linked with the risk of intubation or death $(P>0.05)$. The sensitivity analyses found that lymphocyte count of $<1000$ per $\mathrm{mm} 3$ at hospital admission were still significantly associated with developing ARDS when adjusting for age and male gender ( $\mathrm{HR}, 4.10 ; 95 \% \mathrm{Cl}, 2.40$ 7.10), and oxygenation index (OI) ratio $<150$ were more likely to predict the intubation/death after age adjustment (HR, 2.50; 95\% Cl, 1.17-5.30).

Conclusion: The SARS-CoV-2-caused ARDS was not the typical ARDS according to Berlin criteria. The alive patients with IMV had been intubated earlier since ARDS diagnosis than those who had not survived. We identified male gender and abnormal laboratory indices associated with the ARDS but were not linked with the intubation/death. Sensitivity analysis concluded lymphocyte count of $<1000$ per mm3 could predict ARDS while Ol ratio less than 150 could predict intubation/death.

\section{Introduction}

Since the COVID-19 outbroke in December 2019 in Wuhan of China, it has rapidly developed into a worldwide pandemic, with more than 129 million patients infected which resulted in over 2 million deaths until March 2021 (1). The data published suggest that 5-20\% of COVID-19 patients develop severe illness that is characterized by acute respiratory distress syndrome (ARDS), the mainstay of treatment for which is mechanical ventilation (24). However, the mortality has been reported ranging from $30-80 \%$ in the COVID-19 patients who progressed into invasive mechanical ventilation (IMV) $(2,4)$.

The systematic review on risk factors with ARDS reported lacking robust data of risk factors to prevent COVID-19 and identified an urgent need for high quality research in this area (5). The previous studies generally suffered from unclear definition, small sample sizes, and a lack of adjustment for confounders (5). Until now, the risk factors leading to the intubation or death have not been well delineated $(4,6)$. As those patients with ARDS who progressed on intubation and mechanical ventilation had higher deaths $(2,4)$, identifying the relevant risk factor associated with the intubation or death in the population could change the worst outcomes waiting for global populations fully vaccinated. Additionally, the views that COVID-19 causing an "atypical" ARDS remain different 
and have been discussed $(7,8)$. Comparably large studies are really required to conclude that if COVID-19-related ARDS has different features.

To make full use of the past valuable medical records and improve the future clinical outcomes for the pandemic particularly in several countries, we retrospectively compared the clinical features between COVID-19 patients with ARDS and those without ARDS, and between subgroups of intubation and never intubation in COVID-19 patients from two hospitals of Hubei of China. We analysed the initial intubation time between survivors and non-survivors who were given IMV. We then studied the risk factors for COVID-19 ARDS and the ARDS patients who progressed into intubation or death.

\section{Methods}

\section{Study design and participants}

This retrospective cohort study included adult COVID-19 inpatients ( $\geq 18$ years old) from 26 January 2020 to 1 April 2020 treated at General Hospital of Central Theatre Command of PLA in Wuhan (a tertiary hospital) and at People's Hospital of Honghu (a community hospital) in Jingzhou, a city close to Wuhan, in Hubei province, China. COVID-19 was diagnosed based on the World Health Organization (WHO)'s technical guidance on COVID19 , including confirmation with reverse transcription polymerase chain reaction (RT-PCR) (9).

The COVID-19-related ARDS was diagnosed according to the Berlin criteria published in JAMA: "development of acute and bilateral pulmonary infiltrates and hypoxemia $\left(\mathrm{PaO}_{2} / \mathrm{FIO}_{2} \leq 300 \mathrm{mmHg}\right.$ or $\mathrm{SpO}_{2} / \mathrm{FiO} \mathrm{O}_{2} \leq 315$ as a surrogate if no $\mathrm{PaO}_{2}$ data are available in the study) not primarily due to heart failure or volume overload" (10). In the cohort, the ARDS patients were diagnosed not considering their positive end-expiratory pressure (PEEP) in order to identify the risk factors for the intubation or death earlier. Patients were screened and included by reviewing their electronic medical records and laboratory results. We excluded COVID-19 patients who were not confirmed by the RT-PCR testing, or pneumonia caused by other pathogens, such as bacteria, fungi, mycoplasma or chlamydia.

The study was approved by the Chinese Ethics Committee of Registering Clinical Trials (ChiECRCT20200113), and the requirement for informed consent was waived because of the retrospective study design. All methods were performed in accordance with the Strengthening the Reporting of Observational Studies in Epidemiology (STROBE) Statement (11).

\section{Data collection}

We reviewed the electronic medical records, laboratory results and radiographic reports of all admitted COVID-19 patients. Using a modified version of the standardized case report form developed by the International Severe Acute Respiratory and Emerging Infection Consortium and WHO (12), we extracted data on the demographic characteristics, medical history, comorbidities, illness onset date, initial symptoms, vital signs on admission and other data such as the severity of pneumonia graded by physicians. We also extracted the results of routine hematological, biochemical and inflammation-related indices obtained within 72 hours of hospital admission. treatments (antiviral drugs, antibiotics, glucocorticoids and vasopressor agents) or advanced therapies (intensive care unit (ICU) care, continuous renal-replacement therapy (CRRT), prone position ventilation and extracorporeal membrane oxygenation (ECMO); complications with death and other clinical outcomes. Further, 
we collected information on ARDS diagnosis, including chest imaging; $\mathrm{FiO}_{2}, \mathrm{PaO}_{2}$ or $\mathrm{SpO}_{2}$ values to calculate oxygenation index (OI); oxygen supplement modalities; intubation date; IMV duration; Reports of chest radiographs were obtained, and any factors associated with ARDS and acute respiratory failure warranting intubation in the cohort or with death were recorded.

\section{Outcomes}

The outcomes were ARDS development and intubation or death at hospital.

\section{Statistical analysis}

Continuous data are expressed as medians with interquartile ranges (IQRs). Categorical data are presented as counts ( $\mathrm{n}$ ) with percentages (\%). Missing data were not imputed. We used Kruskal-Wallis rank test and Fisher's exact test to compare the results between ARDS and non-ARDS patients of COVID-19, and between ARDS patients who were never intubated and these who were intubated. Bivariate cox proportional hazard ratio (HR) models were used to determine HRs and 95\% Cls for the risk factors associated with the ARDS or the development from ARDS to intubation/death. Sensitivity analysis yielding adjusted HRs for the popular concerned predictors of lymphopenia and oxygenation index ratio less than 150 were conducted by using multivariate cox proportional HR model. Due to the explorative nature of the study, no formal hypotheses and the sample size calculation were proposed, and we included the maximum number of COVID-19 patients from the two hospitals. $P$ values less than 0.05 were considered significant. All analyses were performed in SPSS (version 25, IBM Corp, Armonk, NY, USA) and Stata (version 16; Stata Corp, College Station, TX, USA) and RStudio (Version 1.3.1056, R Foundation for Statistical Computing).

\section{Results}

\section{Demographics, characteristics, and clinical outcomes}

From 26 January to 1 April 2020, 338 patients with COVID-19 confirmed by laboratory findings or otherwise clinical diagnosis were treated at the two hospitals in Hubei province of China. We included 333 patients, of which 65 subjects developed ARDS, and we excluded 5 cases due to negative/no laboratory testing or insufficient data. The details of enrolment are provided in Fig. 1.

Compared with patients without ARDS, patients with ARDS were older $(P<0.001)$ and trended higher $B M I(P=$ 0.055), and more patients with ARDS were over 65 years $(P=0.015)$ and males $(P=0.008)($ Table 1$)$. Median days from symptoms onset to hospital admission were shorter in ARDS than non-ARDS patients $(P=0.012)$. However, more patients with ARDS presented with initial symptoms of fever $(P=0.028)$, cough $(P=0.009)$, fatigue $(P=0.050)$, expectoration $(P<0.001)$, palpitation and chest distress $(P<0.001)$, and at least three symptoms $(P<0.001)$ compared with those who did not develop ARDS. Patients who progressed into ARDS had higher breath rate $(P=0.008)$, more comorbidities $(P=0.008)$, and severest pneumonia graded by physicians $(P$ $=0.008$ ) on admission than patients not progressing into ARDS. All subjects were found to have bilateral infiltrate in lung in the severe illness of COVID-19 compared to $15.2 \%$ in the non-severe patients $(P<0.001)$. For treatment, the significantly higher percentages of patients with ARDS received general or advanced therapies, including antivirus therapy $(P=0.002)$, antibiotic $(P<0.001)$, glucocorticoid $(P<0.001)$, ICU-level care $(P<0.001)$, prone positioning $(P<0.001)$, CRRT $(P<0.001)$, and ECMO $(P<0.001)$. At last, when compared with patients who 
did not happen ARDS, patients with the severe illness further progressed more on IMV due to failure of noninvasive respiratory support $(P<0.001)$, stayed longer in hospital $(P=0.012)$, and died more $(P<0.001)$. No patient in non-ARDS group occurred severe complications compared to the 13 in ARDS group had acute respiratory failure $(P<0.001)$ and acute circulatory failure $(P<0.001)$, and the 7 occurred Shock/MODS $(P<$ 0.001). 
Table 1

Characteristics and outcomes between COVID-19 patients with ARDS and without ARDS and between the ARDS subgroup of intubation and never intubation

\begin{tabular}{|c|c|c|c|c|c|c|}
\hline \multirow[t]{3}{*}{ Study variables } & \multicolumn{3}{|l|}{ All patients } & \multicolumn{3}{|c|}{ Patients with ARDS } \\
\hline & \multicolumn{3}{|c|}{$\begin{array}{l}\text { Value, no./total no. (\%) or median (IQR) (n } \\
=333 \text { ) }\end{array}$} & \multicolumn{3}{|c|}{$\begin{array}{l}\text { Value, no./total no. }(\%) \text { or median } \\
(\text { IQR })(n=65)\end{array}$} \\
\hline & $\begin{array}{l}\text { Without ARDS } \\
(n=268)\end{array}$ & $\begin{array}{l}\text { With ARDS } \\
(n=65)\end{array}$ & $\begin{array}{l}P \\
\text { value }\end{array}$ & $\begin{array}{l}\text { Intubation } \\
(n=20)\end{array}$ & $\begin{array}{l}\begin{array}{l}\text { Never } \\
\text { Intubation }\end{array} \\
(n=45)\end{array}$ & $\begin{array}{l}P \\
\text { value }\end{array}$ \\
\hline
\end{tabular}

Age

\begin{tabular}{|c|c|c|c|c|c|c|}
\hline median, IQR & $59.0(43.0-68.0)$ & $\begin{array}{l}67.0(56.0- \\
78.0)\end{array}$ & $\begin{array}{l}< \\
0.001\end{array}$ & $\begin{array}{l}71.5(65.5- \\
79.5)\end{array}$ & $\begin{array}{l}61.0(55.0- \\
77.0)\end{array}$ & 0.094 \\
\hline$\geq 65$ years & 100/268 (37.3\%) & $\begin{array}{l}35 / 65 \\
(53.8 \%)\end{array}$ & 0.015 & $\begin{array}{l}15 / 20 \\
(75.0 \%)\end{array}$ & $\begin{array}{l}19 / 45 \\
(42.2 \%)\end{array}$ & 0.015 \\
\hline Male sex & $141 / 268$ (37.3\%) & $\begin{array}{l}46 / 65 \\
(70.8 \%)\end{array}$ & 0.008 & $\begin{array}{l}12 / 20 \\
(60.0 \%)\end{array}$ & $\begin{array}{l}34 / 45 \\
(75.6 \%)\end{array}$ & 0.203 \\
\hline
\end{tabular}

\section{$\mathrm{BMI}^{*}$}

\begin{tabular}{|c|c|c|c|c|c|}
\hline median, IQR & $23.4(21.5-25.5)$ & $\begin{array}{l}24.4(22.4- \\
26.7)(n=52)\end{array}$ & 0.055 & $\begin{array}{l}23.5(21.5- \\
27.2)(n= \\
18)\end{array}$ & $\begin{array}{l}24.6(23.4- \\
26.7)(n= \\
32)\end{array}$ \\
\hline
\end{tabular}

\begin{tabular}{|c|c|c|c|c|c|c|}
\hline$\geq 25$ & $86 / 268(32.1 \%)$ & $21 / 52$ (40\%) & 0.246 & $\begin{array}{l}7 / 18 \\
(38.9 \%)\end{array}$ & $\begin{array}{l}13 / 32 \\
(40.6 \%)\end{array}$ & 0.904 \\
\hline
\end{tabular}

$\begin{array}{lllllll}\text { Smoke history } \quad 22 / 268(8.2 \%) & 7 / 65(10.8 \%) & 0.511 & 2 / 20(10 \%) & \begin{array}{l}5 / 45 \\ (11.1 \%)\end{array} & 1.000\end{array}$

$\begin{array}{lllllll}\text { Alcohol used } & 23 / 268(8.6 \%) & 9 / 65(13.8 \%) & 0.196 & 1 / 20(5 \%) & \begin{array}{l}8 / 45 \\ (17.8 \%)\end{array} & 0.255\end{array}$

\begin{tabular}{|c|c|c|c|c|c|}
\hline $\begin{array}{l}\text { Days from } \\
\text { symptoms onset to } \\
\text { hospital admission }\end{array}$ & $10.0(5.0-15.0)$ & $\begin{array}{l}8.0(5.0- \\
10.0)\end{array}$ & 0.012 & $\begin{array}{l}7.5(5.0- \\
10.0)\end{array}$ & $\begin{array}{l}9.0(5.0- \\
11.0)\end{array}$ \\
\hline
\end{tabular}
(median, IQR)

\section{Initial symptoms}

\begin{tabular}{lllllll} 
Fever & $191 / 268(71.3 \%)$ & $\begin{array}{l}55 / 65 \\
(84.6 \%)\end{array}$ & 0.028 & $\begin{array}{l}18 / 20 \\
(90.0 \%)\end{array}$ & $\begin{array}{l}37 / 45 \\
(82.2 \%)\end{array}$ & 0.711 \\
Cough & & & & & \\
& $142 / 268(53.0 \%)$ & $\begin{array}{l}46 / 65 \\
(70.8 \%)\end{array}$ & 0.009 & $\begin{array}{l}15 / 20 \\
(75.0 \%)\end{array}$ & $\begin{array}{l}31 / 45 \\
(68.9 \%)\end{array}$ & 0.617 \\
\hline
\end{tabular}

* Data regarding the BMI were missing for 28 of 333 patients $(8.4 \%)$.

† All patients first were on noninvasive oxygen support when admitted at hospital.

‡ Data summarized from the patients admitted into ICU.

Abbreviations: IQR, interquartile range, OI: Oxygenation Index, ECMO: Extracorporeal membrane oxygenation, MODS: Multiple Organ Dysfunction Syndrome. 


\begin{tabular}{|c|c|c|c|c|c|c|}
\hline \multirow{3}{*}{$\begin{array}{l}\text { Study variables } \\
\text { Short of breath }\end{array}$} & \multirow{2}{*}{\multicolumn{3}{|c|}{$\begin{array}{l}\text { All patients } \\
\text { Value, no./total no. (\%) or median (IQR) (n } \\
=333 \text { ) }\end{array}$}} & \multirow{2}{*}{\multicolumn{3}{|c|}{$\begin{array}{l}\text { Patients with ARDS } \\
\text { Value, no./total no. (\%) or median } \\
\text { (IQR) }(\mathrm{n}=65)\end{array}$}} \\
\hline & & & & & & \\
\hline & $142 / 268(53.0 \%)$ & $\begin{array}{l}27 / 65 \\
(41.5 \%)\end{array}$ & 0.098 & $\begin{array}{l}12 / 20 \\
(60.0 \%)\end{array}$ & $\begin{array}{l}17 / 45 \\
(37.8 \%)\end{array}$ & 0.096 \\
\hline Fatigue & $56 / 268(20.9 \%)$ & $\begin{array}{l}21 / 65 \\
(32.3 \%)\end{array}$ & 0.050 & $\begin{array}{l}11 / 20 \\
(55.0 \%)\end{array}$ & $\begin{array}{l}10 / 45 \\
(22.2 \%)\end{array}$ & 0.009 \\
\hline Expectoration & $41 / 268(15.3 \%)$ & $\begin{array}{l}17 / 65 \\
(26.2 \%)\end{array}$ & $\stackrel{<}{0.001}$ & $\begin{array}{l}7 / 20 \\
(35.0 \%)\end{array}$ & $\begin{array}{l}10 / 45 \\
(22.2 \%)\end{array}$ & 0.279 \\
\hline $\begin{array}{l}\text { Palpitation and chest } \\
\text { distress }\end{array}$ & $23 / 268$ (8.6\%) & $\begin{array}{l}17 / 65 \\
(26.2 \%)\end{array}$ & $\begin{array}{l}< \\
0.001\end{array}$ & $\begin{array}{l}7 / 20 \\
(35.0 \%)\end{array}$ & $\begin{array}{l}10 / 45 \\
(22.2 \%)\end{array}$ & 0.279 \\
\hline $\begin{array}{l}\text { Number of } \\
\text { complaints } \geq 3\end{array}$ & $113 / 268(42.2 \%)$ & $\begin{array}{l}44 / 65 \\
(67.7 \%)\end{array}$ & $<.001$ & $\begin{array}{l}18 / 20 \\
(90.0 \%)\end{array}$ & $\begin{array}{l}26 / 45 \\
(57.8 \%)\end{array}$ & 0.011 \\
\hline \multicolumn{7}{|l|}{$\begin{array}{l}\text { Vital signs on } \\
\text { admission fever }(\geq \\
\left.37.2^{\circ} \mathrm{C}\right)\end{array}$} \\
\hline $\begin{array}{l}\text { Breath rate (median, } \\
\text { IQR) }\end{array}$ & $19.0(18.0-20.0)$ & $\begin{array}{l}20.0(20.0- \\
24.0)\end{array}$ & 0.008 & $\begin{array}{l}20.0(20.0- \\
22.0)\end{array}$ & $\begin{array}{l}21.0(20.0- \\
24.0)\end{array}$ & 0.641 \\
\hline $\begin{array}{l}\text { Heart rate (median, } \\
\text { IQR) }\end{array}$ & $\begin{array}{l}90.0(80.0- \\
108.0)\end{array}$ & $\begin{array}{l}85.0(79.0- \\
99.0)\end{array}$ & 0.340 & $\begin{array}{l}88.0(80.0- \\
106.0)\end{array}$ & $\begin{array}{l}85.0(79.0- \\
96.0)\end{array}$ & 0.889 \\
\hline SBP (median, IQR) & $\begin{array}{l}126.5(117.5- \\
137.0)\end{array}$ & $\begin{array}{l}135.0(120.0- \\
141.0)\end{array}$ & 0.249 & $\begin{array}{l}145.5 \\
(140.0- \\
154.0)\end{array}$ & $\begin{array}{l}124.0 \\
(116.0- \\
135.0)\end{array}$ & 0.004 \\
\hline DBP (median, IQR) & $79.5(70.0-87.5)$ & $\begin{array}{l}79.0(71.0- \\
86.0)\end{array}$ & 0.849 & $\begin{array}{l}85.0(84.0- \\
86.0)\end{array}$ & $\begin{array}{l}76.0(70.0- \\
84.0)\end{array}$ & 0.131 \\
\hline MAP (median, IQR) & $\begin{array}{l}96.0(86.7- \\
102.0)\end{array}$ & $\begin{array}{l}99.3(86.7- \\
105.0)\end{array}$ & 0.360 & $\begin{array}{l}103.5 \\
(101.3- \\
115.7)\end{array}$ & $\begin{array}{l}94.7(86.7- \\
101.0)\end{array}$ & 0.021 \\
\hline \multicolumn{7}{|l|}{$\begin{array}{l}\text { Severity of } \\
\text { pneumonia graded } \\
\text { by physicians }\end{array}$} \\
\hline Mild & $28 / 260(10.8 \%)$ & $2 / 65(3.1 \%)$ & & $0 / 20(0.0 \%)$ & $2 / 45$ (4.4\%) & \\
\hline Severe & $212 / 260(81.5 \%)$ & $\begin{array}{l}18 / 65 \\
(27.7 \%)\end{array}$ & & $\begin{array}{l}3 / 20 \\
(15.0 \%)\end{array}$ & $\begin{array}{l}15 / 45 \\
(33.3 \%)\end{array}$ & \\
\hline Critical & $20 / 260(7.7 \%)$ & $\begin{array}{l}45 / 65 \\
(68.3 \%)\end{array}$ & $<.001$ & $\begin{array}{l}17 / 20 \\
(85.0 \%)\end{array}$ & $\begin{array}{l}28 / 45 \\
(62.2 \%)\end{array}$ & 0.217 \\
\hline
\end{tabular}

* Data regarding the BMI were missing for 28 of 333 patients (8.4\%).

† All patients first were on noninvasive oxygen support when admitted at hospital.

₹ Data summarized from the patients admitted into ICU.

Abbreviations: IQR, interquartile range, OI: Oxygenation Index, ECMO: Extracorporeal membrane oxygenation, MODS: Multiple Organ Dysfunction Syndrome. 


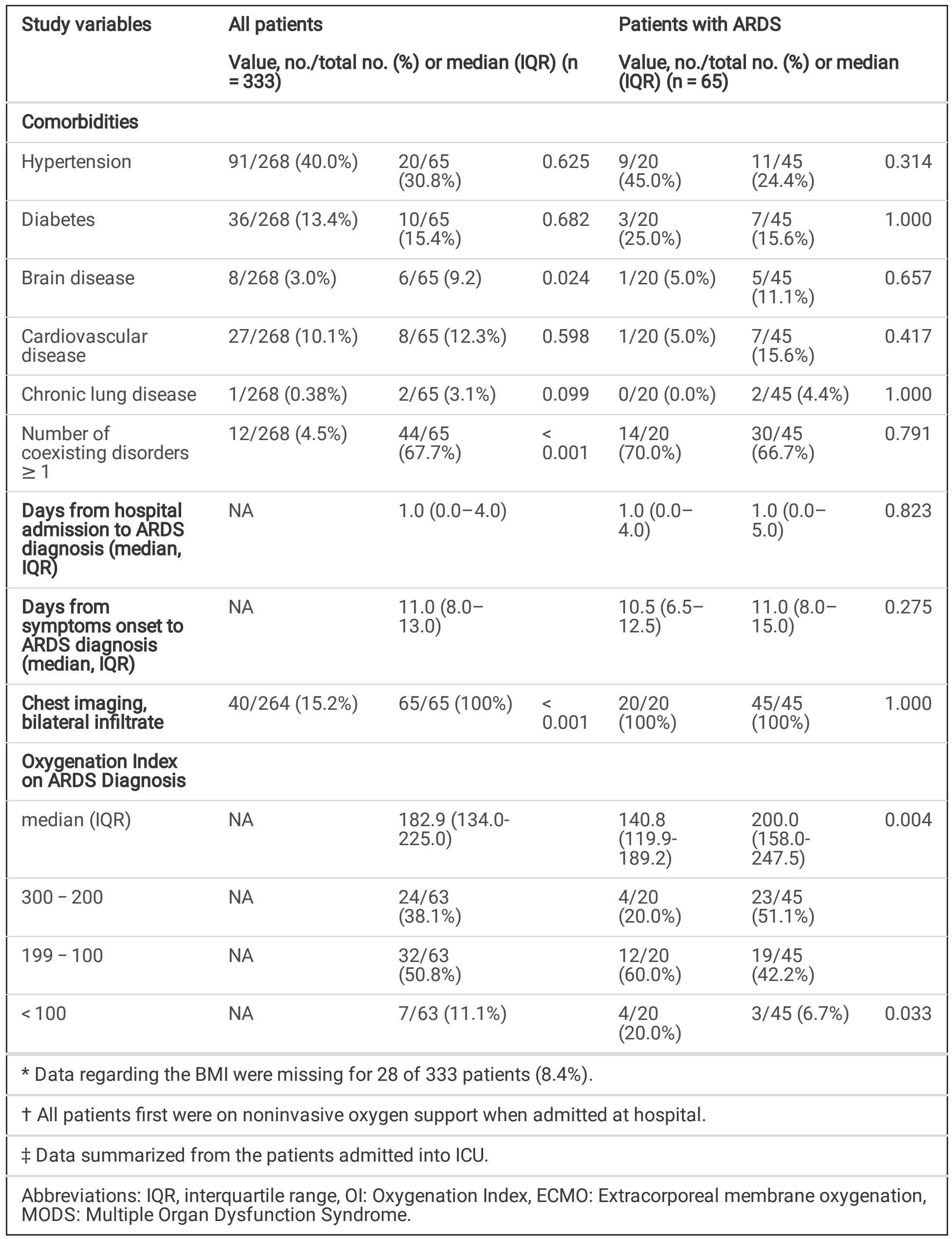




\section{Study variables \\ All patients \\ Value, no./total no. (\%) or median (IQR) (n $=333$ )}

\section{Treatments}

Antiviral therapy

$220 / 268(82.1 \%)$

$63 / 65(95.2 \%) \quad 0.002$

$82 / 268(48.8 \%)$

Intravenous

antibiotic

Glucocorticoid

(methylprednisolone)

Vasopressor

Admission to ICU

$12 / 268(4.5 \%)$

$0 / 268(0.0 \%)$

$7 / 268(2.6 \%)$

$0 / 268(0.0 \%)$

$60 / 65$

$(92.3 \%)$

$\begin{array}{ll}< & 20 / 20 \\ 0.001 & (100 \%)\end{array}$

$51 / 65$ (7.

$8.5 \%)$

$<\quad 20 / 20$

$0.001 \quad(100 \%)$

$16 / 65$

$(24.6 \%)$

$<\quad 10 / 20$

$0.001 \quad(50.0 \%)$

$29 / 65$

(44.6\%)

$<\quad 15 / 20$

$0.001 \quad(75.0 \%)$

$8 / 65(12.3 \%)$

$<\quad 8 / 20$

$0.001 \quad(40.0 \%)$

$6 / 65(9.2 \%)<0 / 20$

$0.001 \quad(30.0 \%)$

Continuous renal-
replacement therapy

ECMO

0/268 (0.0\%)

$0 / 268(0.0 \%)$

$4 / 65(6.2 \%)$
$20 / 20$

(100\%)

$43 / 45$

$(95.3 \%)$

$40 / 45$

$(88.4 \%)$

$31 / 45$

$(67.4 \%)$

$6 / 45$

$(14.0 \%)$

$14 / 45$

$(27.9 \%)$

$0 / 45(0.0 \%)$

0.001

$0 / 45(0.0 \%)<$

0.001

$5 / 20$

(25.0\%)

0.012

$0 / 45(0.0 \%) \quad 0.002$

$0.001 \quad(25.0 \%)$

\section{Outcomes}

\begin{tabular}{|c|c|c|c|c|c|c|}
\hline $\begin{array}{l}\text { Failure of } \\
\text { noninvasive } \\
\text { respiratory support }\end{array}$ & $0 / 268(0.0 \%)$ & $\begin{array}{l}20 / 65 \\
(31.7 \%)\end{array}$ & $<.001$ & $\begin{array}{l}20 / 20 \\
(100 \%)\end{array}$ & $0 / 45(0.0 \%)$ & $<001$ \\
\hline $\begin{array}{l}\text { Median ICU stay } \\
\text { (day, IQR) } \neq\end{array}$ & $7.0(6.0-12.0)$ & $\begin{array}{l}15.0(10.0- \\
20.0)\end{array}$ & 0.075 & $\begin{array}{l}16.0(10.0- \\
21.0)\end{array}$ & $\begin{array}{l}11.0(4.0- \\
16.0)\end{array}$ & 0.131 \\
\hline $\begin{array}{l}\text { Median Hospital stay } \\
\text { (day, IQR) }\end{array}$ & $17.0(12.0-24.0)$ & $\begin{array}{l}22.0(13.0- \\
34.0)\end{array}$ & 0.012 & $\begin{array}{l}25.0(16.0- \\
35.5)\end{array}$ & $\begin{array}{l}22.0(12.0- \\
31.0)\end{array}$ & 0.227 \\
\hline Death in hospital & $0 / 268(0.0 \%)$ & $\begin{array}{l}24 / 65 \\
(36.9 \%)\end{array}$ & $<.001$ & $\begin{array}{l}14 / 20 \\
(70.0 \%)\end{array}$ & $\begin{array}{l}10 / 45 \\
(22.2 \%)\end{array}$ & $<001$ \\
\hline Death of mild ARDS & NA & $5 / 26(19.2 \%)$ & & $3 / 4(75.0 \%)$ & $2 / 23(8.7 \%)$ & 0.013 \\
\hline $\begin{array}{l}\text { Death of moderate } \\
\text { ARDS }\end{array}$ & NA & $\begin{array}{l}13 / 32 \\
(40.6 \%)\end{array}$ & & 7/12(58.3\%) & $6 / 19(31.6 \%)$ & 0.141 \\
\hline $\begin{array}{l}\text { Death of severe } \\
\text { ARDS }\end{array}$ & NA & $6 / 7(85.7 \%)$ & & $4 / 4(100.0 \%)$ & $2 / 3(66.7 \%)$ & .429 \\
\hline
\end{tabular}

* Data regarding the BMI were missing for 28 of 333 patients (8.4\%).

† All patients first were on noninvasive oxygen support when admitted at hospital.

‡ Data summarized from the patients admitted into ICU.

Abbreviations: IQR, interquartile range, Ol: Oxygenation Index, ECMO: Extracorporeal membrane oxygenation, MODS: Multiple Organ Dysfunction Syndrome. 


\begin{tabular}{|c|c|c|c|c|c|c|}
\hline Study variables & All patic & & & Patients & RDS & \\
\hline & $\begin{array}{l}\text { Value, } n \\
=333 \text { ) }\end{array}$ & (\%) or median ( & R) (n & $\begin{array}{l}\text { Value, } n c \\
(\text { IQR })(n=\end{array}$ & I no. (\%) or me & \\
\hline Complications & & & & & & \\
\hline $\begin{array}{l}\text { Acute respiratory } \\
\text { failure }\end{array}$ & $0 / 268$ & $\begin{array}{l}13 / 65 \\
(20.0 \%)\end{array}$ & $<0.001$ & $\begin{array}{l}5 / 20 \\
(25.0 \%)\end{array}$ & $2 / 45(4.4 \%)$ & 0.025 \\
\hline $\begin{array}{l}\text { Acute circulatory } \\
\text { failure }\end{array}$ & $0 / 268$ & $\begin{array}{l}13 / 65 \\
(20.0 \%)\end{array}$ & $<.001$ & $\begin{array}{l}7 / 20 \\
(35.0 \%)\end{array}$ & $\begin{array}{l}6 / 45 \\
(13.3 \%)\end{array}$ & 0.044 \\
\hline Shock/MODS & $0 / 268$ & $7 / 65$ (10.8\%) & $<.001$ & $\begin{array}{l}5 / 20 \\
(25.0 \%)\end{array}$ & $2 / 45(4.4 \%)$ & 0.025 \\
\hline * Data regarding & were & f 333 patie & $3.4 \%)$. & & & \\
\hline † All patients first & noni & support wh & dmitt & thospita & & \\
\hline ‡ Data summarize & $m$ the pat & ed into ICU. & & & & \\
\hline
\end{tabular}

In the subgroup of COVID-19 patients with ARDS, more patients who progressed on IMV were over 65 years $(P=$ $0.015)$, reported fatigue $(P=0.009)$ and at least three symptoms at admission $(P=0.011)$, and had higher SBP $(P=0.004)$ or MAP levels $(P=0.021)$ with admission fever compared with patients who were never intubated (Table 1). The median oxygenation index of intubation patients at ARDS diagnosis were 140.8 (IQR, 119.9-189.2) compared to 200.0 (IQR, 158.0-247.5) of patients without intubation $(P=0.004)$, and higher percentage of intubation patients presented with moderate or severe ARDS at first than patients not on intubation $(P=0.033)$. In addition, patients intubated were more likely to be treated with glucocorticoid $(P=0.003)$ and admitted into ICU $(P=0.012)$, and to receive prone positioning $(P<0.001)$, CRRT $(P<0.001)$, or ECMO $(P=0.002)$ comparing with patients not intubated during hospitalization. A higher proportion of patients intubated developed acute respiratory $(P=0.025)$, circulatory failure $(P=0.044)$, Shock/MODS $(P=0.025)$, and finally died in hospital $(P<$ 0.001). The morality rate was particularly higher in the mild ARDS who received IMV $(P=0.013)$ than those did not.

Laboratory indices tested at admission found that compared with patients without ARDS, for patients with ARDS, the value of liver damage indices (AST $P=0.003$, ALT $P=0.024$, Albumin $P<0.001, A / G P<0.001$ ), heart injury indices (LDH and cTnI $P<0.001$ ), pancreas injury indices (glucose $P<0.001$ ) inflammation-related indices (ESR, CRP and PCT $P<0.001$ ), coagulation function indices (D-Dimer, PT and INR P<0.001), kidney dysfunction indices (CRE and GFR $P<0.001)$ and blood sodium levels were elevated $(P<0.001)$ (Table 2). However, in ARDS patients, blood cell counts of WBC $(P<0.001)$, neutrophil $(P<0.001)$ and lymphocyte $(P<0.001)$ and potassium levels $(P=0.041)$ were significantly decreased. ARDS patients demonstrated immune system destruction indicated by extremely lower median values of lymphocyte count per mm3 (670, IQR 430-1040) and multiple organ injuries suggested by abnormal median values of A/G ratio (1.13, IQR 0.95-1.39), LDH (371.5, IQR 227.0519.0), blood glucose (7.9, IQR 6.0-10.5), and D-Dimer (0.6, IQR 0.2-2.4) levels. In the subgroup analysis, there was no significant difference of laboratory indices between the patients intubated and the patients never 
intubated. Nevertheless, ARDS patients on IMV trended lower cell counts of WBC, neutrophil, and lymphocyte, and elevated markers related to the damages of liver, pancreas, and kidney than the patients never intubated. 
Table 2

Initial laboratory indices between COVID-19 patients with ARDS and without ARDS and between the ARDS subgroup of intubation and never intubation

\section{Laboratory indices on All patients ARDS admission}

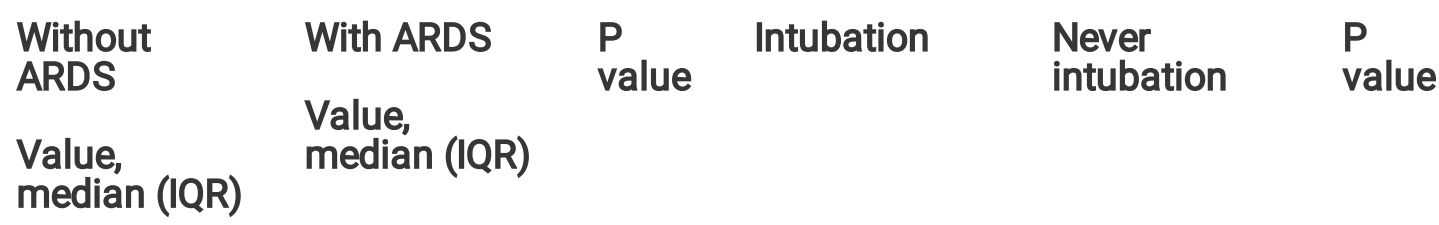

\section{Hematologic}

\begin{tabular}{|c|c|c|c|c|c|c|}
\hline WBC count $/ \mathrm{mm} 3$ & $\begin{array}{l}5300(4200- \\
6400)\end{array}$ & $\begin{array}{l}7340(4700- \\
10700)\end{array}$ & $\begin{array}{l}< \\
0.001\end{array}$ & $\begin{array}{l}6450(4350- \\
101500 .)\end{array}$ & $\begin{array}{l}7340(4810- \\
11500)\end{array}$ & 0.418 \\
\hline $\begin{array}{l}\text { Neutrophil count } \\
/ \mathrm{mm} 3\end{array}$ & $\begin{array}{l}3280(2380- \\
4360)\end{array}$ & $\begin{array}{l}6320(3440- \\
9150)\end{array}$ & $\begin{array}{l}< \\
0.001\end{array}$ & $\begin{array}{l}5170(3365- \\
8985)\end{array}$ & $\begin{array}{l}6490(3490- \\
10590)\end{array}$ & 0.706 \\
\hline $\begin{array}{l}\text { Lymphocyte count } \\
\text { /mm3 }\end{array}$ & $\begin{array}{l}1260(915- \\
1665)\end{array}$ & $\begin{array}{l}670(430- \\
1040)\end{array}$ & $\begin{array}{l}< \\
0.001\end{array}$ & $\begin{array}{l}635(400- \\
795)\end{array}$ & $\begin{array}{l}740(460- \\
1080)\end{array}$ & 0.149 \\
\hline Platelet count /mm3 & $\begin{array}{l}202(157- \\
256)\end{array}$ & $\begin{array}{l}199(146- \\
236)\end{array}$ & 0.259 & $\begin{array}{l}211(119- \\
223)\end{array}$ & $\begin{array}{l}197(154- \\
261)\end{array}$ & 0.4 \\
\hline Hemoglobin, g/dL & $\begin{array}{l}12.6(11.6- \\
13.7)\end{array}$ & $\begin{array}{l}13.1(11.6- \\
14.0)\end{array}$ & 0.185 & $\begin{array}{l}13.0(11.7- \\
13.7)\end{array}$ & $\begin{array}{l}13.2(11.6- \\
14.6)\end{array}$ & 0.367 \\
\hline
\end{tabular}

\section{Biochemical}

\begin{tabular}{|c|c|c|c|c|c|c|}
\hline TBIL, $\mu \mathrm{mol} / \mathrm{L}$ & $\begin{array}{l}11.6(8.6- \\
14.9)\end{array}$ & $\begin{array}{l}13(10.1- \\
18.3)\end{array}$ & 0.069 & $\begin{array}{l}15.0(11.1- \\
17.7)\end{array}$ & $\begin{array}{l}12.0(9.8- \\
18.3)\end{array}$ & 0.323 \\
\hline AST, U/L & $\begin{array}{l}27.0(22.0- \\
38.5)\end{array}$ & $\begin{array}{l}39.0(25.0- \\
50.0)\end{array}$ & 0.002 & $\begin{array}{l}37.5(26.5- \\
50.0)\end{array}$ & $\begin{array}{l}39.0(23.0- \\
50.0)\end{array}$ & 0.793 \\
\hline$A L T, U / L$ & $\begin{array}{l}22.0(15.0- \\
36.0)\end{array}$ & $\begin{array}{l}27.0(20.0- \\
35.0)\end{array}$ & 0.024 & $\begin{array}{l}25.0(18.0- \\
29.0)\end{array}$ & $\begin{array}{l}28.0(20.0- \\
43.0)\end{array}$ & 0.176 \\
\hline Total protein, g/L & $\begin{array}{l}64.7(61.2- \\
70.5)\end{array}$ & $\begin{array}{l}64.0(60.7- \\
68.3)\end{array}$ & 0.156 & $\begin{array}{l}64.8(63.0- \\
68.5)\end{array}$ & $\begin{array}{l}62.6(59.9- \\
68.3)\end{array}$ & 0.179 \\
\hline Albumin, g/L & $\begin{array}{l}39.2(35.8- \\
41.7)\end{array}$ & $\begin{array}{l}34.1(31.2- \\
37.5)\end{array}$ & $\begin{array}{l}< \\
0.001\end{array}$ & $\begin{array}{l}34.2(30.5- \\
36.9)\end{array}$ & $\begin{array}{l}34.1(31.3- \\
38.0)\end{array}$ & 0.994 \\
\hline
\end{tabular}

* Data regarding the cTnl were missing for 2 ARDS patients who were never intubated.

† Data regarding the Glucose were missing for 1 ARDS patient who were never intubated.

‡ Data regarding both the Potassium and Sodium were missing for 6 ARDS patients, with 4 at never intubation group, 1 at early intubation group, and 1 at late intubation group.

$\S$ Data regarding the PTs were missing for 1 ARDS patient who were never intubated.

|| Data regarding the ESR were missing for 6 ARDS patients who were never intubated.

Abbreviations: IQR, interquartile range, WBC: White Blood Cell; TBIL: Total bilirubin, AST: aspartate transaminase, ALT: alanine aminotransaminase, LDH: lactate dehydrogenase, GFR: glomerular filtration rate, PT: prothrombin time, INR: international normalized ratio, ESR: Erythrocyte Sedimentation Rate. 


\begin{tabular}{|c|c|c|c|c|c|c|}
\hline Laboratory indices on & All patients & & & ARDS & & \\
\hline Albumin/Globulin & $\begin{array}{l}1.49(1.27- \\
1.66)\end{array}$ & $\begin{array}{l}1.13(0.95- \\
1.39)^{(2)}\end{array}$ & $<.001$ & $\begin{array}{l}1.13(0.86- \\
1.25)^{(}\end{array}$ & $\begin{array}{l}1.16(0.96- \\
1.39)^{(2)}\end{array}$ & 0.344 \\
\hline LDH, unit/L & $\begin{array}{l}210.0(171.0- \\
266.0)\end{array}$ & $\begin{array}{l}371.5(227.0- \\
519.0)\end{array}$ & $<0.001$ & $\begin{array}{l}384.0(218.0- \\
519.0)\end{array}$ & $\begin{array}{l}361.0(241.0- \\
525.0)\end{array}$ & 0.927 \\
\hline $\mathrm{cTnl}, \mathrm{ng} / \mathrm{mL}^{*}$ & $\begin{array}{l}0.01(0.01- \\
0.01)\end{array}$ & $\begin{array}{l}0.02(0.01- \\
0.04)\end{array}$ & $<0.001$ & $\begin{array}{l}0.02(0.01- \\
0.03)\end{array}$ & $\begin{array}{l}0.01(0.01- \\
0.06)\end{array}$ & 0.953 \\
\hline Glucose, mmol/L† & $5.8(5.2-6.7)$ & $7.9(6.0-10.5)$ & $<001$ & $\begin{array}{l}7.8(6.1- \\
10.2)\end{array}$ & $7.9(6.0-10.7)$ & 0.739 \\
\hline Creatinine, $\mu \mathrm{mol} / \mathrm{L}$ & $\begin{array}{l}65.0(53.0- \\
76.0)\end{array}$ & $\begin{array}{l}74.2(59.0- \\
93.0)\end{array}$ & $<001$ & $\begin{array}{l}74.4(66.5- \\
107.5)\end{array}$ & $\begin{array}{l}74.2(57.0- \\
92.5)\end{array}$ & 0.685 \\
\hline $\mathrm{GFR}, \mathrm{ml} / \mathrm{min} / 1.73 \mathrm{~m}^{2}$ & $\begin{array}{l}100.0(89.0- \\
113.0)\end{array}$ & $\begin{array}{l}89.0(62.0- \\
104.0)\end{array}$ & $<0.001$ & $\begin{array}{l}72.5(60.0- \\
94.5)\end{array}$ & $\begin{array}{l}93.0(68.0- \\
108.0)\end{array}$ & 0.120 \\
\hline Potassium, mmol/L‡ & $3.8(3.5-4.1)$ & $3.9(3.7-4.3)$ & 0.041 & $4.0(3.7-4.1)$ & $3.9(3.6-4.3)$ & 0.967 \\
\hline Sodium, mmol/Lł & $\begin{array}{l}140.6(138.8- \\
142.3)\end{array}$ & $\begin{array}{l}137.8\left(134.8^{-}\right. \\
139.3)\end{array}$ & $<001$ & $\begin{array}{l}137.6(135.0- \\
139.3)\end{array}$ & $\begin{array}{l}137.9(134.8- \\
139.3)\end{array}$ & 0.882 \\
\hline D-Dimer, mg/L & $0.1(0.1-0.3)$ & $0.6(0.2-2.4)$ & $<0.001$ & $0.4(0.2-2.3)$ & $0.7(0.2-2.4)$ & 0.776 \\
\hline PT, s§ & $\begin{array}{l}11.8(11.3- \\
12.6)\end{array}$ & $\begin{array}{l}13.1(11.9- \\
14.7)\end{array}$ & $<0.001$ & $\begin{array}{l}13.1(11.7- \\
13.9)^{(1.7}\end{array}$ & $\begin{array}{l}13.2(12.2- \\
14.8)\end{array}$ & 0.339 \\
\hline INR & $1.1(1.0-1.2)$ & $1.1(1.1-1.2)$ & $<.001$ & $1.2(1.1-1.2)$ & $1.1(1.1-1.2)$ & 0.438 \\
\hline $\mathrm{ESR}, \mathrm{mm} / \mathrm{h} \|$ & $\begin{array}{l}22.0(11.0- \\
50.0)\end{array}$ & $\begin{array}{l}62.5(31.0- \\
81.0)\end{array}$ & $<0.001$ & $\begin{array}{l}66.5(30.5- \\
84.5)\end{array}$ & $\begin{array}{l}60.5(39.0- \\
80.0)\end{array}$ & 0.832 \\
\hline $\begin{array}{l}\text { C-Reactive Protein, } \\
\text { mg/L }\end{array}$ & $\begin{array}{l}10.0(3.5- \\
26.3)\end{array}$ & $\begin{array}{l}59.1(20.1- \\
108.6)\end{array}$ & $<0.001$ & $\begin{array}{l}72.0(38.2- \\
110.6)\end{array}$ & $\begin{array}{l}48.2(19.5- \\
108.6)\end{array}$ & 0.410 \\
\hline Procalcitonin, $\mu \mathrm{g} / \mathrm{L}$ & $\begin{array}{l}0.04(0.03- \\
0.07)\end{array}$ & $\begin{array}{l}0.12(0.05- \\
0.38)\end{array}$ & $<0.001$ & $\begin{array}{l}0.21(0.07- \\
0.50)\end{array}$ & $\begin{array}{l}0.10(0.05- \\
0.35)\end{array}$ & 0.438 \\
\hline \multicolumn{7}{|c|}{ * Data regarding the cTnl were missing for 2 ARDS patients who were never intubated. } \\
\hline \multicolumn{7}{|c|}{ † Data regarding the Glucose were missing for 1 ARDS patient who were never intubated. } \\
\hline \multicolumn{7}{|c|}{$\begin{array}{l}\text { ₹ Data regarding both the Potassium and Sodium were missing for } 6 \text { ARDS patients, with } 4 \text { at never } \\
\text { intubation group, } 1 \text { at early intubation group, and } 1 \text { at late intubation group. }\end{array}$} \\
\hline \multicolumn{7}{|c|}{$\S$ Data regarding the PTs were missing for 1 ARDS patient who were never intubated. } \\
\hline \multicolumn{7}{|c|}{ || Data regarding the ESR were missing for 6 ARDS patients who were never intubated. } \\
\hline $\begin{array}{l}\text { Abbreviations: IQR, in } \\
\text { transaminase, ALT: al } \\
\text { PT: prothrombin time }\end{array}$ & $\begin{array}{l}\text { artile range, } \\
\text { aminotrans }\end{array}$ & $\begin{array}{l}\text { White Blo } \\
\text { inase, LDH: }\end{array}$ & $\begin{array}{l}\text { II; TBI } \\
\text { e deh deh }\end{array}$ & $\begin{array}{l}\text { Total bilirubi } \\
\text { rogenase, GF }\end{array}$ & $\begin{array}{l}\text { aspartate } \\
\text { nerular filtra }\end{array}$ & rate, \\
\hline
\end{tabular}




\section{High mortality of COVID-19 ARDS patients with intubation and the initial time of intubation}

COVID-19 ARDS patients requiring intubation showed a high mortality at 70.0\% (Table 1). However, we found the survivors had been intubated earlier since ARDS diagnosis than the non-survivors, with a median of 5.5 and IQR of 4.0-7.0 days versus a median of 11.5 and IQR 6.0-14.0 days $(P<0.001)$ (Fig. 2).

\section{Risk factors associated with ARDS and intubation/death in COVID-19 patients}

Univariate cox proportion hazard models showed that age and age over 65 years were associated with the development of ARDS (HR, 1.04; $95 \% \mathrm{Cl}, 1.01-1.06, \mathrm{HR}, 2.24 ; 95 \% \mathrm{Cl}, 1.18-4.28)$ and the intubation/death (HR, $1.03 ; 95 \% \mathrm{Cl}, 1.01-1.06, \mathrm{HR}, 3.07 ; 95 \% \mathrm{Cl}, 1.32-7.16$ ) (Table 3). Other factors including male gender and abnormal laboratory indices related to the development of ARDS were not found to be associated with the intubation/death. However, the number of symptom complaints equal and more than three was associated with higher likelihood of progressing into intubation/death $(\mathrm{HR}, 4.74 ; 95 \% \mathrm{Cl}, 1.65-13.66)$. Patients on lower $\mathrm{Ol}$ ratio at ARDS diagnosis ( $\mathrm{HR}, 0.99 ; 95 \% \mathrm{Cl}, 0.98-1.00)$ and patients with Ol ratio less than $200(\mathrm{HR}, 3.05 ; 95 \% \mathrm{Cl}, 1.24-$ 7.46), 150 ( $\mathrm{HR}, 3.00 ; 95 \% \mathrm{Cl}, 1.45-6.17)$, or $100(\mathrm{HR}, 2.54 ; 95 \% \mathrm{Cl}, 1.03-6.25)$ were more likely to require intubation or die in hospital. Sensitivity analysis showed that when adjusting for age and gender, COVID-19 patients with lymphocyte count of $<1000$ per $\mathrm{mm} 3$ at admission were still significantly associated with developing ARDS (HR, 4.10; 95\% Cl, 2.40-7.10). When adjusting for age, ARDS patients with lower Ol ratio less than 150 at ARDS diagnosis were also more likely to need the intubation or happen the death $(\mathrm{HR}, 2.50 ; 95 \% \mathrm{Cl}$, 1.17-5.30) (Table S1). 
Table 3

Risk factors associated with ARDS and ARDS progressing on Intubation or Death

Variables

ARDS $(n=65)$

Intubation or Death $(n=30)$

$\operatorname{HR}(95 \% \mathrm{Cl})$

$P$ value $\quad H R(95 \% \mathrm{Cl})$

$P$ value

Clinical characteristics

Age

$1.04(1.01-1.06)$

0.001

$1.03(1.01-1.06)$

0.019

Age $(\geq 65)$ years

$2.24(1.18-4.28)$

0.014

$3.07(1.32-7.16)$

0.009

Gender (male vs female)

$2.08(1.03-4.20)$

0.040

$0.70(0.32-1.49)$

0.352

Number of complaints $(\geq 3)$

$1.50(0.77-2.95)$

0.236

$4.74(1.65-13.66)$

0.004

Hypertension

$1.03(0.53-2.02)$

0.928

$1.61(0.77-3.35)$

0.204

Number of coexisting disorders $(\geq 1)$

$1.15(0.57-2.36)$

0.693

$1.82(0.74-4.50)$

0.192

Oxygenation Index

\begin{tabular}{lllll} 
Values & NA & NA & $0.99(0.98-1.00)$ & 0.001 \\
\hline$<200$ & NA & NA & $3.05(1.24-7.46)$ & 0.015 \\
\hline$<150$ & NA & NA & $3.00(1.45-6.17)$ & 0.003 \\
$<100$ & NA & NA & $2.54(1.03-6.25)$ & 0.042
\end{tabular}

\section{Laboratory findings}

Lymphocyte count /mm3 (< 1000)

$4.72(2.34-9.51)$

$<0.001 \quad 1.58(0.64-3.87)$

0.319

Albumin, $\mathrm{g} / \mathrm{L}(<32)$

$3.36(1.70-6.66)$

0.001

$0.88(0.38-2.07)$

0.775

Albumin/Globulin (<1.5)

$10.02(3.08-32.60)$

$<0.001$

$0.89(0.34-2.34)$

0.818

LDH, unit/L (> 280)

$5.44(2.78-10.64) \quad<0.001$

$1.10(2.51-0.35)$

0.810

$\mathrm{cTnl}, \mathrm{ng} / \mathrm{mL}(>0.04)$

$3.80(1.91-7.55)$

$<0.001$

$0.84(0.36-1.95)$

0.678

Glucose, $\mathrm{mmol} / \mathrm{L}(>7.1)$

$4.33(2.27-8.25)$

$<0.001$

$1.46(0.64-2.92)$

0.425

Creatinine, $\mu \mathrm{mol} / \mathrm{L}(>110)$

$3.88(1.62-9.31)$

0.002

$0.54(0.19-1.54)$

0.247

* Data regarding the cTnl were missing for 2 ARDS patients who were dead.

† Data regarding the Glucose were missing for 1 ARDS patient who were never intubation or not dead.

₹ Data regarding the PTs were missing for 1 ARDS patient who were never intubated or not dead.

$\S$ Data regarding the ESR were missing for 6 ARDS patients who were never intubated or not dead.

Abbreviations: HR; hazard ratio; WBC: White Blood Cell; TBIL: Total bilirubin, AST: aspartate transaminase, ALT: alanine aminotransaminase, LDH: lactate dehydrogenase, GFR: glomerular filtration rate, PT: prothrombin time, INR: international normalized ratio, ESR: Erythrocyte Sedimentation Rate. 


\begin{tabular}{|c|c|c|c|c|}
\hline \multirow{2}{*}{$\begin{array}{l}\text { Variables } \\
\text { GFR, } \mathrm{ml} / \mathrm{min} / 1.73 \mathrm{~m} 2(<80)\end{array}$} & \multicolumn{2}{|l|}{ ARDS $(n=65)$} & \multicolumn{2}{|c|}{ Intubation or Death $(n=30)$} \\
\hline & $3.64(1.91-6.93)$ & $<0.001$ & $1.18(0.58-2.43)$ & 0.648 \\
\hline Sodium, mmol/L (< 135) & $3.84(1.90-7.75)$ & $<0.001$ & $0.88(0.38-2.05)$ & 0.766 \\
\hline D-Dimer, mg/L (>0.5) & $3.99(2.10-7.57)$ & $<0.001$ & $0.81(0.39-1.67)$ & 0.569 \\
\hline PT, s (> 13.5) & $5.55(2.93-10.49)$ & $<0.001$ & $0.69(0.33-1.46)$ & 0.337 \\
\hline INR (> 1.1) & $10.02(3.08-32.60)$ & $<0.001$ & $1.18(0.56-2.47)$ & 0.659 \\
\hline $\mathrm{ESR}, \mathrm{mm} / \mathrm{h}(>20)$ & $6.80(2.09-22.12)$ & 0.001 & $1.33(0.32-5.60)$ & 0.696 \\
\hline C-Reactive Protein, mg/L (> 10) & $7.47(2.92-19.1)$ & $<0.001$ & $0.71(0.25-2.06)$ & 0.533 \\
\hline Procalcitonin, $\mu \mathrm{g} / \mathrm{L}(>0.1)$ & $4.39(2.32-8.30)$ & $<0.001$ & $0.74(0.36-1.52)$ & 0.418 \\
\hline \multicolumn{5}{|c|}{ * Data regarding the cTnl were missing for 2 ARDS patients who were dead. } \\
\hline \multicolumn{5}{|c|}{ † Data regarding the Glucose were missing for 1 ARDS patient who were never intubation or not dead. } \\
\hline \multicolumn{5}{|c|}{ ¥ Data regarding the PTs were missing for 1 ARDS patient who were never intubated or not dead. } \\
\hline \multicolumn{5}{|c|}{$\S$ Data regarding the ESR were missing for 6 ARDS patients who were never intubated or not dead. } \\
\hline $\begin{array}{l}\text { Abbreviations: HR; hazard rati } \\
\text { ALT: alanine aminotransamin } \\
\text { prothrombin time, INR: interna }\end{array}$ & $\begin{array}{l}\text { Blood Cell; TBIL: } \\
\text { ate dehydrogenas } \\
\text { ized ratio, ESR: EI }\end{array}$ & $\begin{array}{l}\text { jilirut } \\
: \text { gloi } \\
\text { yte S }\end{array}$ & $\begin{array}{l}\text { ST: aspartate tr } \\
\text { ar filtration rate } \\
\text { intation Rate. }\end{array}$ & se, \\
\hline
\end{tabular}

\section{Discussion}

In this study, we observed that the ARDS caused by COVID-19 was not the typical ARDS defined by the Berlin criteria due to more than 1-week onset time, multiple organs injuries, and higher mortality. We found that the survivors who were on IMV had been intubated earlier since ARDS diagnosis than the non-survivors. We reported the different risk factors between COVID-19 patients who developed ARDS after admission and who progressed from ARDS to intubation or death.

Older age or age over 65 years was the most consistently reported risk factors for patients who develop ARDS, as concluded by previous investigations (13). Interestingly, we found the hazards ratio of age over 65 years associated with the intubation or death was larger than the ratio of this factor with ARDS, which suggests that the risk for worse clinical outcomes markedly increased once the elder patients developed ARDS. The reason could be the accelerating decline of immune response $(14,15)$. To prevent the ARDS development for the elder patients shall reduce the worse outcomes. Another different finding compared with the studies published was that males faced a significantly greater risk of developing ARDS. However, for the typical ARDS caused by other factors, females were found to be more likely to develop the disease than males (16). One possible explanation was the protection of $\mathrm{X}$ chromosome and sex hormones which played an important role in innate and adaptive immunity made the females less susceptible to coronavirus infection than men, resulting in the less development of the critical disease (17). 
The two-hospital cohort found that the COVID-19 subjects with abnormal laboratory indices at admission were more possibly to progress into ARDS. The lymphopenia, which is defined as the counts less than 1000 counts/mm3, strongly predicted the ARDS for COVID-19 patients. Even adjusting for age and male gender, the lymphopenia was associated with fourfold risk of ARDS. Lymphocytes express the ACE2 receptor and may be a direct target of SARS-CoV-2 infection, and an increase of pro-inflammatory cytokines in COVID-19, especially IL6 , could induce further lymphocyte reduction (18). When the patient's laboratory indicator showed PCT $>0.1$ $\mathrm{ng} / \mathrm{mL}, \mathrm{ESR}>20 \mathrm{~mm} / \mathrm{h}$ or C-Reactive Protein $>10 \mathrm{mg} / \mathrm{L}$, there was a higher risk of progression to the critical illness. That may support the corticosteroids use to prevent the risk of ARDS, particularly for the elder patients, due to alleviating the systemic inflammation (19). The positive associations between albumin $<32 \mathrm{~g} / \mathrm{L}, \mathrm{LDH}>$ $280 \mathrm{U} / \mathrm{L}, \mathrm{cTnl}, \mathrm{ng} / \mathrm{mL}>0.04$, creatinine $>110 \mu \mathrm{mol} / \mathrm{L}$ or glucose $>7.1 \mathrm{mmol} / \mathrm{L}$ and the ARDS indicated that the liver, heart, kidney or pancreas dysfunctions had been involved, and corresponding therapies should be taken in time to prevent the further deterioration of the COVID-19. When the D-dimer $>0.5 \mathrm{mg} / \mathrm{L}$ meant the high risk of the progression of the COVID-19 to ARDS, the approaches to alter the hypercoagulability of blood, reduce the risk of thrombosis, and improve the ischemia and hypoxia shall be implemented early (18).

No laboratory indicators predicting the need of intubation or death suggests different pathophysiological pathways between ARDS development and hypoxemic respiratory failure/death in COVID-19. The ARDS patients presenting with symptoms $\geq 3$ were more likely to be intubated or die at hospital. The clinicians might monitor these ARDS patients more closely to implement therapies in time even though the patients did not suffer any coexisting disease. Additionally, the analysis revealed that COVID-19 patients with lower OI ratios at ARDS diagnosis were more likely to develop hypoxemic respiratory failure necessitating IMV and had higher risk to die. The COVID-19 Guidelines of Europe (20), USA (21), and Canada (22) have not yet provided any recommendation related to intubation. Our findings that the decreasing OI ratio in ARDS patients was highly associated with hypoxemic respiratory failure necessitating the intubation and the risk of in-hospital death may supported the early intubation criteria of the COVID-19 Management -- patients with an $\mathrm{SpO}_{2}$ value of less than $93 \%$ of the room air and a OI ratio of less than $300 \mathrm{mmHg}$ should be prepared for intubation if there are indications (23). The sensitivity analysis showed that the Ol ratio less than 150 well predicted the need of intubation and death when adjusting for age. The threshold might be considered as a caution to the deterioration of COVID-19 patients to facilitate the early treatment.

The study complements the existing small studies on the clinical features of ARDS caused by COVID-19 with a comparably large sample. The longer median onset days (11.0, IQR, 8.0-13.0), which exceeds 1-week limit of typical ARDS (10), should alert physicians to monitor the COVID-19 patients longer for adequate treatment. The mortality rates of moderate and severe COIVID-19 ARDS were higher than the corresponding data of $32 \%$ and $45 \%$ of the typical ARDS (24). One possible explanation we thought is the silent hypoxemia that the patient had severe hypoxemia but was asymptomatic, which would lead to delay treatment $(25,26)$. Therefore, from the perspective of treatment, an adjusted classification of ARDS severity that can correctly identify COVID-19 patients who require a timely therapy for has been always needed (7). Most importantly, the ARDS caused by COVID-19 demonstrated injuries in multiple organs, including the heart, the liver, the kidney, and the pancreas, which was supported by the fundamental research that this new coronavirus can attack the tissues of other organs in addition to the respiratory tract (15). At the two-hospital cohort, 78.5\% patients with ARDS were given systemic corticosteroids to alleviate hyperinflammation. However, guidance regarding corticosteroids use for COVID-19 is still mixed (27). Some issued a weak recommendation (28), but others issued a weak

Page $18 / 25$ 
recommendation against the use of corticosteroids for COVID-19 and ARDS (29). Nevertheless, the latest publication of three randomized trials of corticosteroids and the prospective meta-analysis have strengthened confidence and shifted usual care of COVID-19-related ARDS to include corticosteroids (27).

An interesting result in this cohort was that the survivors with IMV were intubated earlier than those with IMV who did not survive. We noticed the non-survivor group had the median intubation days at 11.5, which exceed one week after ARDS diagnosis or hospital admission. By contrast, we observed the patients intubated within one week after ICU admission or ARDS diagnosis demonstrated much lower mortalities in our cohort, in other hospitals of China (6), and some places of USA (30). To intubate the COVID-19 patients within one week since critical illness development seemed to result in a lower mortality rate. However, because we were not sure the two hospital used the same intubation criteria at that time, and we did not have the specific values when they were progressed into intubation, we could not say these ARDS patients had survived due to comparable early intubation. Even nowadays, the definition of what constitutes "early" is still a challenge and requires necessary investigations for COVID-19. As Shoemaker et al.'s study (23) highlights the importance of timely stopping an enlarging oxygen debt using effective oxygenation and ventilation therapies because there is a close association between the oxygen debt accumulated over 48 hour and the chance of survival in patients undergoing high-risk surgery and ICU admission afterward.

The strengths of the study are as follows: the study cohort included a comparably large sample size from two hospitals in two cities of Hubei province, which increases the robustness and generalizability of the findings; reported HRs for the risk factors and did the sensitivity analysis for the popular concerned risk factors associated with ARDS and ARDS progressing on IMV or death. However, the study has some limitations. First, owing to limited medical resources, only patients with relatively severe COVID-19 were hospitalized during this period. As such, this study may have included disproportionately more patients with poor outcomes. There may also be a selection bias when identifying factors that influence the clinical outcomes. Second, it is a retrospective cohort conducted at two hospitals. As is known, it was hard to perform a perspective cohort when pandemic happened and developed everywhere. As we have sparse new COVID-19 cases now, we hope our new findings can encourage a larger prospective cohort study or even randomly controlled trials in other regions who are experiencing the outbreak and in the urgent need to adjust the strategy.

\section{Conclusions}

In conclusion, the SARS-CoV-2-caused ARDS was not the typical ARDS for which the management strategies should be modulated accordingly. The survival patients had been intubated earlier since ARDS diagnosis than those who had not survived, which may propose the issue of timing of intubation. Lymphopenia predicted the ARDS and OI ratio less than 150 predicted the intubation/death. The male gender and all the abnormal laboratory indices associated with the ARDS but were not linked with the intubation or death, suggesting different pathophysiological processes at the two stages.

\section{Abbreviations}

COVID-19: coronavirus disease 2019; IMV: invasive mechanical ventilation; CFR: case fatality rate; OI: oxygenation index; CRRT: continuous renal-replacement therapy; ECMO: extracorporeal membrane oxygenation; WHO: World Health Organization; ICU: intensive care unit; Pa02/FiO2: arterial partial pressure of oxygen/fraction 
of inspired oxygen; Sp02/FiO2: oxygen saturation by pulse oximetry/fraction of inspired oxygen; PEEP: positive end-expiratory pressure; RRs: risk ratios; IQR: interquartile range; Cl: confidence intervals; SARS-CoV-2: severe acute respiratory syndrome coronavirus 2; BMI: body mass index; ESR: erythrocyte sedimentation rate; GFR: glomerular filtration rate; WBC: white blood cell; TBIL: total bilirubin; AST: aspartate transaminase; ALT: alanine aminotransaminase; LDH: lactate dehydrogenase; GFR: glomerular filtration rate; PT: prothrombin time; INR: international normalized ratio; ESR: erythrocyte sedimentation rate; SBP: systolic blood pressure; DBP: diastolic blood pressure; MAP: mean arterial pressure

\section{Declarations}

\section{Ethics approval and consent to participate}

The study was approved by the Chinese Ethics Committee of Registering Clinical Trials (ChiECRCT20200113), and the requirement for informed consent was waived.

\section{Consent for publication}

Not applicable

\section{Availability of data and materials}

The datasets generated during and analyzed during the current study are not publicly available due to privacy or ethical restrictions but are available from the corresponding author on reasonable request. A proposal with detailed description of study objectives and statistical analysis plan will be needed for evaluation of the reasonability of requests.

\section{Competing interests}

We declare no competing interests.

\section{Funding}

This work was supported by the President Fund of Nanfang Hospital (2018B008) and the National Natural Science Foundation of China (81902009, 81730058, 81671955).

\section{Author Contributions}

JLM and KXL conceived the study and its design and take responsibility for the integrity of the data and accuracy of the analysis. JLM, MZQ, HMS, FZ, DSQ, HY, JN, and YHD organized and entered data. JLM, BSG, SDQ, FZ, HY, and XuD Liu contributed to data analyses. JLM, MZQ, BSG, XuDL, XiaoDL, WFY, BXL, XYS, ShYL, and KXL contributed to data interpretation. JLM and BSG drafted the manuscript. All authors critically revised the drafted manuscript and approve of the submitted manuscript.

\section{Acknowledgments}

We thank all patients involved in the study, residents, and nurses who helped with data collection. We appreciate Prof. Hao Liu for his advice on manuscript improvement. We would like to thank Dr. Hua Jiang, the frontline 
expert of COVID-19 management, for her advice on the data interpretation. We are very thankful for secretary Ms. Jing-Xin Xiao for her assistance in the research conducting.

\section{References}

1. WHO. World Health Organization: Coronavirus Disease (COVID-19) Dashboard; 2020. Cited March 312021. Available from: https://covid19.who.int/.

2. Guan W, Ni Z, Hu Y, Liang W, Ou C, He J, et al. Clinical Characteristics of Coronavirus Disease 2019 in China. N Engl J Med. 2020;382(18):1708-20.

3. Yang X, Yu Y, Xu J, Shu H, Xia J, Liu H, et al. Clinical course and outcomes of critically ill patients with SARSCoV-2 pneumonia in Wuhan, China: a single-centered, retrospective, observational study. The Lancet Respiratory medicine. 2020;8(5):475-81.

4. Cummings MJ, Baldwin MR, Abrams D, Jacobson SD, Meyer BJ, Balough EM, et al. Epidemiology, clinical course, and outcomes of critically ill adults with COVID-19 in New York City: a prospective cohort study. medRxiv : the preprint server for health sciences. 2020.

5. C Carver NJ. Are there risk factors and preventative interventions for acute respiratory distress syndrome (ARDS) in COVID-19? : The Centre for Evidence-Based Medicine develops, promotes and disseminates better evidence for healthcare; 2020. Cited June 8 2020. Available from: https://www.cebm.net/covid-19/are-thererisk-factors-and-preventative-interventions-for-acute-respiratory-distress-syndrome-ards-in-covid-19/.

6. Wang T, Tang C, Chen R, Ruan H, Liang W, Guan W, et al. Clinical Features of Coronavirus Disease 2019 Patients With Mechanical Ventilation: A Nationwide Study in China. Crit Care Med. 2020;48:e809-e12.

7. Li X, Ma X. Acute respiratory failure in COVID-19: is it "typical" ARDS? Critical care (London, England). 2020;24(1):198.

8. Fan E, Beitler JR, Brochard L, Calfee CS, Ferguson ND, Slutsky AS, et al. COVID-19-associated acute respiratory distress syndrome: is a different approach to management warranted? The Lancet Respiratory medicine. 2020;8(8):816-21.

9. Novel coronavirus - China: World Health Organization; 2020. Cited March 31 2021. Available from: http://www.who.int/csr/don/12-january-2020-novel-coronavirus-china/en/.

10. The ARDS Definition Task Force. Acute Respiratory Distress Syndrome: The Berlin Definition. JAMA. 2012;307(23):2526-33.

11. Vandenbroucke JP, von Elm E, Altman DG, Gøtzsche PC, Mulrow CD, Pocock SJ, et al. Strengthening the Reporting of Observational Studies in Epidemiology (STROBE): explanation and elaboration. PLoS medicine. 2007;4(10):e297.

12. ISARIC WHO SARI Case Record Form: The International Severe Acute Respiratory and Emerging Infection Consortium (ISARIC); 2020. Cited May 5 2020. Available from: https://isaric.tghn.org/.

13. Grasselli G, Greco M, Zanella A, Albano G, Antonelli M, Bellani G, et al. Risk Factors Associated With Mortality Among Patients With COVID-19 in Intensive Care Units in Lombardy, Italy. JAMA Internal Medicine. 2020;180(10):1345-55.

14. Wu C, Chen X, Cai Y, Xia Ja, Zhou X, Xu S, et al. Risk Factors Associated With Acute Respiratory Distress Syndrome and Death in Patients With Coronavirus Disease 2019 Pneumonia in Wuhan, China. JAMA Internal Medicine. 2020;180:1-11. 
15. Chowdhury MA, Hossain N, Kashem MA, Shahid MA, Alam A. Immune response in COVID-19: A review. Journal of Infection and Public Health. 2020.

16. Heffernan DS, Dossett LA, Lightfoot MA, Fremont RD, Ware LB, Sawyer RG, et al. Gender and acute respiratory distress syndrome in critically injured adults: a prospective study. J Trauma. 2011;71(4):878-85.

17. Zheng Z, Peng F, Xu B, Zhao J, Liu H, Peng J, et al. Risk factors of critical \& mortal COVID-19 cases: A systematic literature review and meta-analysis. J Infect. 2020;81(2):e16-e25.

18. Huang I, Pranata R. Lymphopenia in severe coronavirus disease-2019 (COVID-19): systematic review and meta-analysis. Journal of Intensive Care. 2020;8(1):36.

19. Villar J, Ferrando C, Martínez D, Ambrós A, Muñoz T, Soler JA, et al. Dexamethasone treatment for the acute respiratory distress syndrome: a multicentre, randomised controlled trial. The Lancet Respiratory medicine. 2020;8(3):267-76.

20. COVID-19 Guidelines: Society of Critical Care Medicine; 2020. Cited June 1 2020. Available from: https://www.sccm.org/SurvivingSepsisCampaign/Guidelines/COVID-19.

21. Coronavirus Disease 2019 (COVID-19) Treatment Guidelines: National Institutes of Health; 2020. Cited June 1 2020. Available from: https://www.covid19treatmentguidelines.nih.gov/whats-new/.

22. Clinical management of patients with moderate to severe COVID-19 - Interim guidance: Government of Canada; 2020. Cited July 28 2020. Available from: https://www.canada.ca/en/publichealth/services/diseases/2019-novel-coronavirus-infection/clinical-management-covid-19.html.

23. Meng L, Qiu H, Wan L, Ai Y, Xue Z, Guo Q, et al. Intubation and Ventilation amid the COVID-19 Outbreak: Wuhan's Experience. Anesthesiology: The Journal of the American Society of Anesthesiologists. 2020;132(6):1317-32.

24. Fanelli V, Vlachou A, Ghannadian S, Simonetti U, Slutsky AS, Zhang H. Acute respiratory distress syndrome: new definition, current and future therapeutic options. J Thorac Dis. 2013;5(3):326-34.

25. Ottestad W, Seim M, Mæhlen JO. COVID-19 with silent hypoxemia. Tidsskrift for den Norske laegeforening : tidsskrift for praktisk medicin, ny raekke. 2020;140(7).

26. Nickson C IJ, Young P. "Silent hypoxaemia” and COVID-19 intubation: Life in the fastlane; 2020. Cited Feb 5 2021. Available from: https://litfl.com/silent-hypoxaemia-and-covid-19-intubation/.

27. Prescott HC, Rice TW. Corticosteroids in COVID-19 ARDS: Evidence and Hope During the Pandemic. JAMA. 2020.

28. Alhazzani W, Møller MH, Arabi YM, Loeb M, Gong MN, Fan E, et al. Surviving Sepsis Campaign: Guidelines on the Management of Critically III Adults with Coronavirus Disease 2019 (COVID-19). Crit Care Med. 2020;48(6):e440-e69.

29. Bhimraj A, Morgan RL, Shumaker AH, Lavergne V, Baden L, Cheng VC, et al. Infectious Diseases Society of America Guidelines on the Treatment and Management of Patients with COVID-19. Clinical infectious diseases : an official publication of the Infectious Diseases Society of America. 2020.

30. Suleyman G, Fadel RA, Malette KM, Hammond C, Abdulla H, Entz A, et al. Clinical Characteristics and Morbidity Associated With Coronavirus Disease 2019 in a Series of Patients in Metropolitan Detroit. JAMA Network Open. 2020;3(6):e2012270.

\section{Figures}



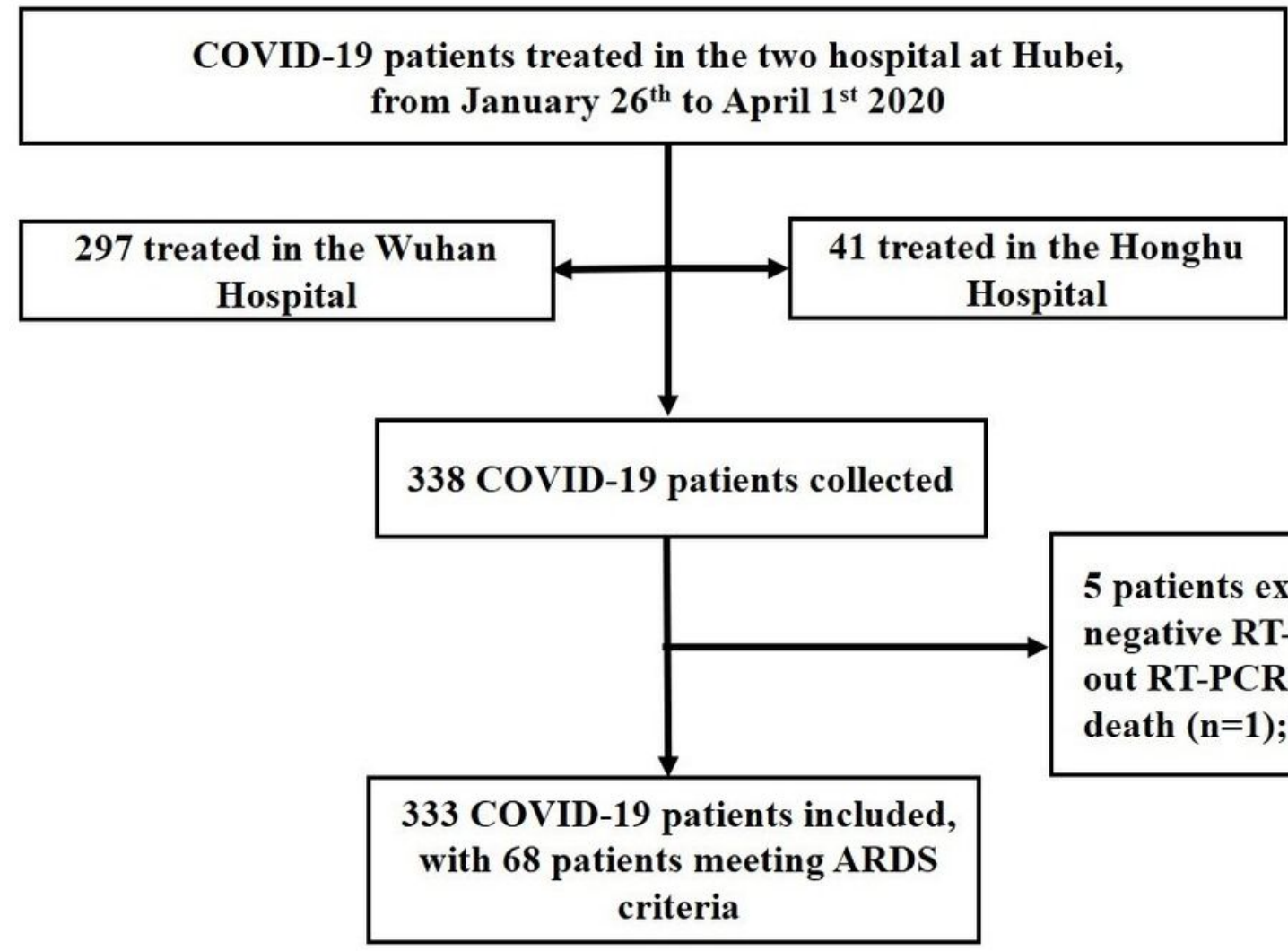

5 patients excluded:

negative RT-PCR twice $(n=3)$; with out RT-PCR testing due to early

death $(n=1)$; insufficient data $(n=1)$

Figure 1

Flowchart of patient's enrolment 
A

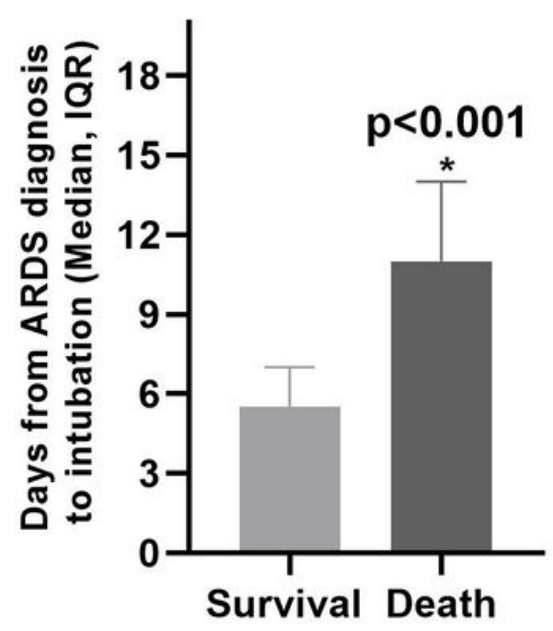

ARDS patients with intubation $(n=20)$

B

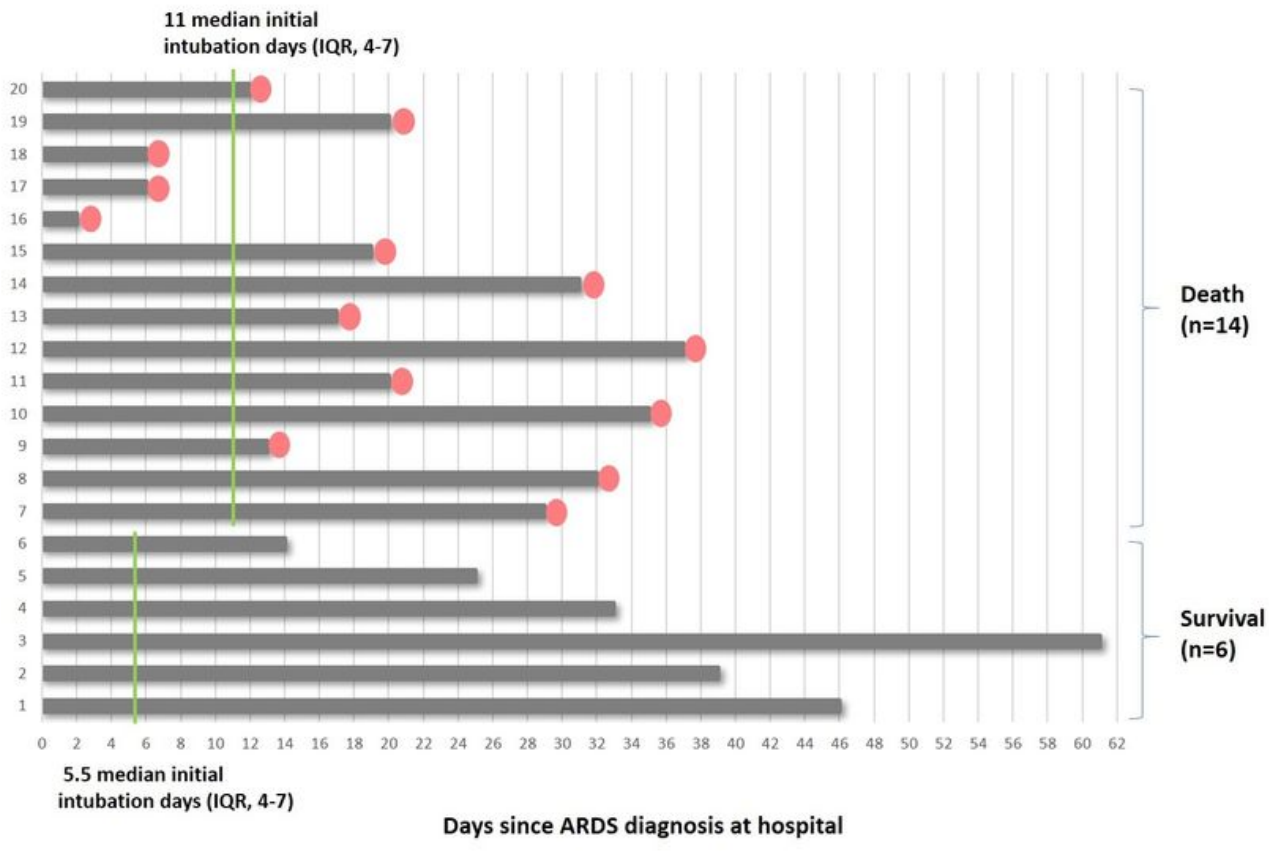

Figure 2

COVID-19 ARDS patients with intubation and the outcomes. A. Median (IQR) days from ARDS diagnosis to intubation between survival and dead patients; $B$. Outcomes of the patients receiving intubation; $P<0.05$. IQR, interquartile range.

\section{Supplementary Files}

This is a list of supplementary files associated with this preprint. Click to download. 
- SupplementaryTable1BMCAnes.docx

Page 25/25 